Revista de Psicología de la PUCP. Vol. XIX, 1, 2001

\title{
El consumo de drogas y el tratamiento en pacientes que realizan actividades delictivas ${ }^{1}$
}

\author{
Manuel Sanabria Carretero²
}

El trabajo se centra en valorar las implicaciones que la actividad delictiva tiene, tanto en el inicio como en la evolución, y los resultados del tratamiento por consumo de drogas. A tal fin, se seleccionó una muestra de 209 pacientes que solicitaron tratamiento. Ellos fueron valorados en las principales áreas objeto de análisis y seguidos durante un año y medio; se realizaron valoraciones de su evolución cada seis meses. Los resultados indican que el paso por la institución terapéutica conlleva una reducción muy significativa de la actividad delictiva, aunque los pacientes con un mayor historial ofrecen más dificultades y una respuesta inicial más pobre; no obstante, en el seguimiento estas diferencias llegan a desaparecer.

Palabras clave: drogadicto, actividad ilegal, tratamiento, seguimiento.

\section{The drug taking and the treatment in patients that are involved in illegal activities}

The study focuses on the assessment of the implications that criminal behavior has, not only at the start of treatment for drug taking but also during its development and results. With this aim in mind a sample of 209 patients who had applied for treatment were selected and assessed in the principal object areas of analysis over a period of a year and a half at six monthly intervals. The results indicate that time spent in a therapeutic centre significantly reduces criminal behaviour. However, those patients with a greater past history present more difficulties and give a poorer initial response, although during the period of follow-up these differences tend to disappear.

Keywords: drug addict, illegal activity, treatment, follow-up.

1. El presente trabajo se ha llevado a cabo en cinco Centros Asistenciales de la Red Gallega de Drogodependencias. La coordinación del mismo corrió a cargo del Departamento de Psicología Clínica y Psicobiología de la Universidad de Santiago y formó parte de un proyecto de investigación más amplio.

2. Doctor en psicología por la Universidad de Santiago de Compostela. Su área de especialización e investigación es la drogodependencia. Ha escrito numerosos artículos sobre ese tema. Ha trabajado en el Programa de Rehabilitación de Drogodependientes Proyecto Hombre, con el que continúa colaborando. Ha desarrollado el Programa de Prevención del Consumo de Drogas CINENSINO. Actualmente trabaja como profesor y psicólogo. C/ Rosalía de Castro, 55 - $4^{\circ} \mathrm{C}$ - 15895 Milladoiro - Santiago de Compostela - Coruña - España. masanabria@teleline.es. 

La problemática delictiva en el campo de las drogodependencias cabe ser abordada desde dos puntos de vista. En primer lugar, puede considerarse un síntoma de la personalidad antisocial, por lo que podría encuadrarse dentro de los trastornos psicológicos asociados al consumo; por otra parte, puede constituir una actividad con fines meramente económicos destinados a la financiación y procura de la droga; en este sentido, dicha práctica reúne, para muchos adictos, características de actividad a full time, de tal manera que puede ser considerada casi como una especie de "profesión" dirigida a la procura y mantenimiento del hábito de consumo.

Sobre el primer aspecto, Gerstley, Alterman, McLellan y Woody (1990) indican que no se puede relacionar, de forma directa, la actividad delictiva con el consumo de drogas. Kandel (1978) y Roelof (1986) precisan que la mitad de los drogodependientes ya la desarrollaban antes de que se iniciaran en el consumo y que el poco aumento puede atribuirse a este. Tampoco los cambios en tipo, frecuencia y cantidad de droga consumida iban parejos a cambios en la actividad delictiva; concretamente, en un período de cinco años de observación, el incremento del consumo no se correspondía con el de delincuencia. Se constató incluso que la actividad delictiva tendía a decrecer con el paso del tiempo.

Así pues y desde este punto de vista, la actividad delictiva desplegada por estos pacientes no responde exclusivamente a necesidades relacionadas con la economía del consumo, sino que habría un factor añadido y referido a características de la personalidad. Una de esas características puede ser la búsqueda de sensaciones, nos referimos con ello a lo atractivo que puede resultar para el sujeto el implicarse en conductas de riesgo y vivir en los límites de la legalidad. En estos casos, la actividad delictiva precede o coexiste con la drogadicción (Byquist, 1999). 
Sin embargo, para otros pacientes la actividad delictiva sí estaría directamente vinculada al consumo y sería posterior a él; existe la creencia de que la toxicomanía constituye un foco de peligrosidad social. Esto ha llevado a establecer bastante causalidad entre consumo de drogas y actividad delictiva. A juicio de Judson y Goldstein (1982), constituiría la principal fuente de ingresos y ocuparía la mayor parte de la actividad del sujeto. Para estos adictos, a mayor actividad delictiva mayor severidad en el consumo de drogas, tendiendo a fluctuar a medida que también lo hace el consumo (Anglin y Speckart, 1988; Dembo et al., 1991; Alemi, Stephens, Llorens y Orris, 1995).

Algunos de estos aspectos fueron aclarados por Hanlon, Nurco, Kinlock y Duszynski (1990). Estos autores estudiaron la trayectoria de 250 sujetos adictos calculando las tasas medias de comisión de delitos para los períodos correspondientes a antes del consumo de drogas, durante la adicción y a lo largo de la abstinencia posterapéutica. Los resultados apuntan a un progresivo descenso de la actividad delictiva a medida que transcurren los sucesivos períodos. Postulan que el aumento de edad puede ser una de las variables explicativas que contribuye a los descensos a medida que el sujeto va madurando.

Parece, sin embargo, que esta progresión no es la misma para todo tipo de drogas. Según el estudio de Dembo et al. (1991), el consumo de marihuana, hachís y cocaína tiene mucha menos implicación en la actividad delictiva que la que tiene la heroína.

Teniendo en cuenta algunos de estos aspectos, nos proponemos estudiar la relación entre la actividad delictiva y el consumo de drogas, así como el peso de aquella variable en la evolución y resultados del tratamiento. Para algunos autores, los pacientes con un amplio historial delictivo suelen tener una recuperación más problemática con resultados más pobres (Pérez Fernández, 1990; Joe, Chastain, Marsh y Simpson, 1990; Dembo et al. 1991; Alemi et al. 1995; Ramsay y Sondhi, 2000). 
El consumo de drogas y el tratamiento en pacientes que realizan actividades dilictivas

Después del tratamiento y a lo largo del seguimiento, si el sujeto ha conseguido tener un control sobre el consumo, cabe esperar que se vea reflejado en la actividad delictiva; sin embargo, esta tendencia no aparece con la misma uniformidad en todos los estudios, ya que cuando se toman como criterio de valoración el número de arrestos, juicios y encarcelamientos pueden aparecer tendencias en alza (Segest, Mygind y Bay, 1989; Sanchez-Carbonell, Brigós y Camí, 1989; Jamoulle, 2000). Una explicación sobre esto apunta a la lentitud y burocratización del sistema judicial por lo que muchos sujetos son detenidos, juzgados o encarcelados por delitos que cometieron hace ya varios años, mantienen en la actualidad un estatus de abstinente $o$ reinsertado y con incidencia del área delictivo-judicial, lo que indica un claro desfase temporal.

\section{La presente investigación}

Con el fin de poder valorar las posibles implicaciones que la actividad delictiva puede tener para los pacientes adictos sometidos a tratamiento, hemos adoptado un diseño longitudinal de panel, seleccionado una muestra a la que hemos seguido desde antes de iniciar el tratamiento (durante él y en el seguimiento) tomando medidas cada seis meses en las áreas objeto de estudio y a lo largo de un año y medio.

\section{Objetivos}

Para corroborar los aspectos anteriores y hacer una estimación del papel que puede desempeñar la actividad delictiva tanto en la drogadicción como en los tratamientos, nos hemos planteado clarificar las siguientes hipótesis:

- La actividad delictiva desplegada por la persona antes de iniciar el tratamiento tiene relación directa con el consumo de drogas.

- Los participantes con puntuaciones más altas en la Batería de Búsqueda de Sensaciones (BBS) tienen un historial delictivo mayor que los pacientes con puntuaciones más bajas. 
- A mayor actividad delictiva antes del tratamiento, cabe esperar resultados más pobres en el tratamiento y seguimiento.

\section{Metodología}

\section{Instrumentos}

Entrevista Estructurada de Recogida de Datos (EERD). Es una adaptación de la utilizada en el proyecto EMETYS (Sánchez-Carbonell, Brigós y Camí, 1989). El instrumento recoge los datos desde lo más general hasta lo más específico. En lo relativo al área de consumo de drogas, se va precisando la información con preguntas que miden la amplitud o duración del consumo y la intensidad del mismo en términos de frecuencia, desde el pasado (un año, tres meses antes) hasta los 30 últimos días.

La Batería de Búsqueda de Sensaciones (BBS). Se relaciona con un rasgo caracterizado por la avidez de estímulos, experiencias variadas y deseo de asumir riesgos para lograr esas experiencias. La escala desarrollada por Zuckerman (1979) se compone de cuarenta ítems distribuídos en cuatro subescalas: emoción y búsqueda de sensaciones, búsqueda de experiencias, desinhibición y susceptibilidad al aburrimiento. Se puede trabajar con las subescalas o con la puntuación total, lo que resulta igualmente discriminante (Luengo, Otero, Romero y Gómez, 1996).

\section{Procedimiento}

La aplicación de los cuestionarios la realizaba el entrevistador que previamente convocaba al paciente, generalmente en el propio centro cuando no era posible en otro lugar previamente acordado $y$, en última instancia, por teléfono. En todos los casos, los entrevistadores eran licenciados en psicología y asistentes sociales a los que se les entrenó previamente y tuvieron un rodaje de una semana para 
familiarizarse con los cuestionarios, sin que estos fueran tenidos en cuenta para el estudio.

Mediante los instrumentos empleados se obtuvo información, en primer lugar, sobre el nivel de consumo y abstinencia de las diferentes sustancias que se distinguieron de entre la heroína, por ser ésta la principal droga que motiva el ingreso y el resto de sustancias ("otras drogas"). En ambos casos se utilizó como criterio la frecuencia del consumo en el último mes, pudiendo oscilar los valores entre cero (ningún consumo) y ocho (más de un consumo diario).

En segundo lugar se elaboró un Índice Global de Adaptación (IGA) que resume en una puntuación que oscila entre 0 y 4 la situación evolutivo-terapéutica del sujeto. Al paciente se le suma un punto por cada una de las condiciones establecidas que cumpla, a saber: no consumir heroína; no consumir otras drogas ni alcohol por encima de los $75 \mathrm{cc}$. de alcohol puro al día en el último mes; en tercer lugar, trabajar o estar matriculado en algún curso; y, por último, no haber cometido actividad ilegal en los últimos tres meses.

En tercer lugar, la Búsqueda de Sensaciones que recogió en una puntuación la suma de los diez ítems de las cuatro subescalas y presentó un formato de respuesta dicotómica.

Finalmente, se registró el número de delitos realizados, con lo que se sumó los valores de once variables que recogen conductas de carácter delictivo (diversos tipos de robo, estafa, falsificación, compra-venta ilegal, prostitución, etc.), así como dos más referidas a detenciones policiales y juicios, todas ellas centradas en el año anterior a la entrevista de inicio.

\section{Participantes}

La muestra se compuso de 209 adictos (83\% varones y $17 \%$ mujeres) con una media de edad de 25 años que acudieron a tratamiento por problemas de consumo en cinco centros de la comunidad gallega. 
Los tratamientos administrados son, en primer lugar, Programas Libres de Drogas, bien en la modalidad ambulatoria o en centros de día, $y$, en segundo lugar, de Mantenimiento con Metadona.

Respecto del área toxicológica, la totalidad de la muestra era consumidora habitual de diversas sustancias, el policonsumo fue la característica más representativa. La sustancia más consumida era la heroína, cuyo consumo es diario para el $72 \%$ de la muestra, seguida del hachís, tranquilizantes y cocaína, consumidas de manera semanal por el $53 \%, 47 \%$ y $41 \%$, respectivamente.

En cuanto al área delictiva, en el momento de acudir al tratamiento la actividad ilegal de mayor incidencia era la venta y distribución de drogas, practicada alguna vez por el $59 \%$ de las personas; cada uno de los participantes se habían iniciado en ella a los 19.3 años (media). La venta ilegal de objetos implica a la mitad de la muestra (50\%), se iniciaron a los 19.5 años; si bien en los últimos tres meses esta actividad ha descendido y se sitúa en el $34 \%$. El robo sin violencia ha sido cometido por el $48 \%$ de la muestra y en idéntico nivel se ha mantenido en el último año, si bien ha descendido en los últimos tres meses al $26 \%$. En el robo con forzamiento han estado implicados el $31 \%$ de las personas y en los tres meses últimos lo han practicado el $12 \%$. Finalmente, el robo de radiocasetes es una infracción cometida por el $28 \%$ y realizada últimamente por el $20 \%$. El robo bajo coacción o a mano armada es el que reviste un descenso más notable, el $12 \%$ lo realizó alguna vez y solo el $7 \%$ lo hizo en los tres meses últimos.

Los delitos de estafa y falsificación tienen una edad de inicio más tardío, aunque a los 20 años ya lo han practicado el $32 \%$ y $14 \%$ respectivamente, se ha reducido al $27 \%$ y $10 \%$ en los últimos meses. Finalmente, el recurso de la prostitución, ligado al consumo de drogas, solo ha sido para el 5\% de la muestra total, de los que el $31 \%$ la continuaban practicando últimamente. En el Cuadro 1 se ofrece resumidamente estos datos. Se puede constatar que los delitos contra la propiedad constituyen, en su conjunto, la actividad ilegal de mayor incidencia. 
El consumo de drogas y el tratamiento en pacientes que realizan actividades dilictivas

\section{Cuadro 1}

Actividad delictiva previa al tratamiento $(n=209)$

\begin{tabular}{|c|c|c|c|c|c|c|c|c|}
\hline \multirow[t]{3}{*}{ Actividad ilegal } & \multirow{3}{*}{$\begin{array}{c}\text { \% Realizada } \\
\text { alguna vez }\end{array}$} & \multirow{3}{*}{$\begin{array}{r}\bar{x} \text { edad } \\
\text { inicio }\end{array}$} & \multicolumn{3}{|c|}{ Último año } & \multicolumn{3}{|c|}{ Últimos 3 meses } \\
\hline & & & & $\%$ & $\%$ & & $\%$ & $\%$ \\
\hline & & & $n$ & $\geq 1$ vez & 0 veces & $n$ & $\geq 1 \mathrm{vez}$ & 0 veces \\
\hline Venta de drogas & 59 & 19.3 & 121 & 64 & 36 & 119 & 46 & 54 \\
\hline Venta de objetos & 50 & 19.5 & 107 & 56 & 44 & 106 & 34 & 66 \\
\hline Robo sin violencia & 48 & 18 & 98 & 48 & 52 & 98 & 26 & 74 \\
\hline Robo con forzamiento & 31 & 18.6 & 63 & 19 & 78 & 62 & 12 & 88 \\
\hline Robo radiocasete & 28 & 18 & 56 & 30 & 68 & 55 & 20 & 80 \\
\hline Robo de coches & 17 & 16 & 36 & 28 & 72 & 36 & 14 & 86 \\
\hline Robo mano armada & 12 & 18 & 26 & 7 & 93 & 26 & 7 & 93 \\
\hline Estafa & 32 & 20 & 65 & 51 & 49 & 64 & 27 & 73 \\
\hline Falsificación & 14 & 20 & 30 & 44 & 56 & 29 & 10 & 89 \\
\hline Prostitución & 5 & 18.2 & 13 & 39 & 61 & 13 & 31 & 69 \\
\hline
\end{tabular}

\section{Resultados}

En primer lugar nos vamos a centrar en la evolución de la actividad delictiva. Para determinar la evolución de esta variable a lo largo de los cuatro períodos de observación realizamos la Figura 1. Como se puede observar, la actividad delictiva sufre un continuo y progresivo descenso desde el momento que el paciente ingresa en tratamiento y se prolonga a lo largo del seguimiento.

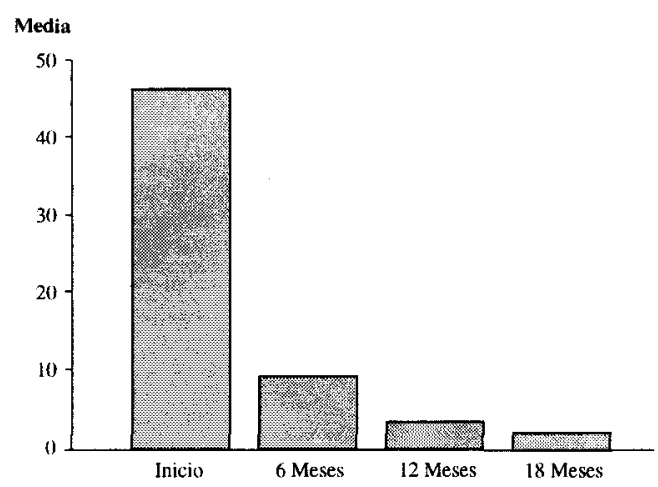

Figura 1. Evolución del número de delitos durante el tratamiento. 
A primera vista, la figura parece ilustrar el efecto benéfico del tratamiento en la reducción de la actividad delictiva, con ello se confirma que existen diferencias significativas entre los sucesivos pases mediante un análisis de varianza de medidas repetidas $\left(\mathrm{F}_{1.208}=95.8 ; \mathrm{p}=.00\right)$. Tal influencia puede distribuirse por dos vías: en primer lugar, la reducción en la tasa de consumo o consecución de la abstinencia como consecuencia de la implicación del adicto en la institución terapéutica puede hacer disminuir la práctica delictiva en aquellos pacientes cuyo consumo era subvencionado por dicha actividad. Por otra parte, el contexto terapéutico supone un ambiente de control en la organización de la vida del paciente con visitas diarias o internado y actividades de ocupación que suponen un distanciamiento de la práctica delictiva. En términos generales, nuestros resultados concuerdan con lo señalado por Hanlon et al. (1990) en tanto apunta a progresivos descensos de la actividad delictiva desde el contacto del paciente con la institución terapéutica.

Para tratar de aclarar la primera de las hipótesis, tendente a valorar la contribución de la actividad delictiva en el consumo de drogas, se llevó a cabo un análisis de regresión múltiple; dicho análisis permitió determinar el valor predictivo de una o varias variables independientes (VI) sobre otra considerada como dependiente (VD) o a predecir. El análisis de regresión, aplicado antes de iniciar el tratamiento, reflejaría las variables que tienen una mayor contribución en la situación adictiva del paciente valorada a través de la frecuencia del consumo de heroína, por ser la droga principal. Este análisis, mediante el método stepwise, va incorporando cada variable independiente hasta seleccionar las que mejor se ajustan a la ecuación de regresión, en nuestro caso el consumo de heroína previo al tratamiento. Dichas variables resultaron ser las referidas al área delictivo-legal y la conflictividad en las relaciones familiares. Ambas variables suman una capacidad predictiva del $10 \%\left(R^{2}=0.10\right)$; aunque es poco, son los únicos coeficientes estadísticamente significativos en tanto quedan fuera de la ecuación de regresión otras veinticuatro variables carentes de valor predictivo significativo. Ver Cuadro 2. 
El consumo de drogas y el tratamiento en pacientes que realizan actividades dilictivas

\section{Cuadro 2}

Variables predictoras respecto del consumo de heroína

\begin{tabular}{|cccccc|}
\hline Variables I. & $B$ & S.E. B & Beta & $T$ & Sig T \\
\hline Actividad delictiva & 0.27 & 0.06 & 0.28 & 4.11 & $\mathbf{0 . 0 0}$ \\
Relaciones famil. conflictivas & 0.24 & 0.11 & 0.15 & 2.13 & $\mathbf{0 . 0 3}$ \\
(Constante) & 2.75 & 0.49 & & 5.55 & 0.00 \\
\hline
\end{tabular}

Así pues, antes de iniciar los tratamientos, la implicación del adicto en actividades de carácter delictivo y el nivel de conflictividad en las relaciones entre los miembros del sistema familiar serían las variables que mejor predicen el consumo de heroína.

Respecto de la segunda hipótesis, pensamos que de existir cierta susceptibilidad de los sujetos con puntuaciones altas en la BBS a buscar y experimentar sensaciones fuertes y de cierto peligro, posiblemente estos aspectos se verán reflejados en el área delictiva ya que muchas de esas conductas reúnen tales características. Efectivamente, al relacionar la puntuación total de la BBS con el historial delictivo de los pacientes, el coeficiente de correlación de Pearson es significativo $(r=0.17 ; p=$ .01 ), lo que quiere decir que los pacientes con puntuaciones más altas en la Batería de Búsqueda de Sensaciones tienden a acumular un mayor número de conductas delictivas en sus historiales. Al dividir la muestra en dos grupos, en función de las puntuaciones bajas y altas de la BBS y compararlos respecto de la actividad delictiva, la $t$ de Student indica que las diferencias entre ambos grupos son estadísticamente significativas. Los 131 sujetos con puntuaciones más altas en la BBS tienen un mayor historial delictivo que aquellos pacientes con puntuaciones inferiores ( $t=$ $3.03, p=.00)$; podría pensarse que estos sujetos tienen una motivación añadida que se recoge en los ítems de la BBS, aspecto que también se ha señalado en alcohólicos (González, Ibáñez y Peñate, 1997).

Así pues, este cuestionario parece resultar interesante en el momento de discriminar a los pacientes adictos con tendencia a implicarse en actividades de carácter delictivo. Posiblemente, para muchos de estos sujetos la 
práctica delictiva se desmarque de las necesidades del consumo y su inicio y evolución no estaría determinado por el uso de sustancias.

En la tercera hipótesis se trata de apreciar si existe alguna relación entre el nivel de implicación delictiva (número de delitos) y las variables dependientes referidas al consumo o abstinencia de heroína, "otras drogas" e IGA. Estas variables fueron relacionadas entre sí, en el Cuadro 3 se muestran los índices de correlación de Pearson hallados. Los coeficientes de los seis meses corresponden a la respuesta al tratamiento y parecen confirmar lo postulado por la hipótesis; el valor positivo del índice nos indica que se trata de una relación directa, es decir, que a mayor actividad delictiva un año antes del tratamiento corresponden mayores tasas de consumo o una menor abstinencia en la etapa del tratamiento. El valor negativo de las $\mathrm{r}$ para el IGA indica que la adaptación tiende a ser peor a medida que la implicación delictiva previa ha sido mayor. A este nivel, nuestros datos coinciden con lo señalado por otros autores que aprecian resultados más pobres en el tratamiento en pacientes con mayor implicación delictiva (Sánchez-Carbonell, Camí y Brigós, 1988; Joe et al., 1990; Dembo et al., 1991; Alemi et al., 1995).

\section{Cuadro 3}

Relaciones entre actividad delictiva previa y resultados del tratamiento

\begin{tabular}{|ccl|}
\hline & Actividad delictiva previa & \\
\hline Consumo heroína & Consumo "otras drogas" & IGA \\
\hline & $\mathbf{6}$ meses & \\
\hline $\boldsymbol{r}=\mathbf{0 . 1 9}$ & $\boldsymbol{r}=\mathbf{0 . 2 6}$ & $\boldsymbol{r}=\mathbf{- 0 . 2 7}$ \\
$p=.01$ & $p=.001$ & $p=.001$ \\
$n=145$ & $n=145$ & $n=151$ \\
\hline & $\mathbf{1 2}$ meses & \\
\hline$r=0.03$ & $\mathrm{r}=0.01$ & $r=-0.03$ \\
$p=.72$ & $p=.85$ & $p=.73$ \\
$n=123$ & $n=123$ & $n=123$ \\
\hline & $\mathbf{1 8}$ meses & \\
\hline$r=0.06$ & $\boldsymbol{r}=\mathbf{0 . 2 4}$ & $r=-0.12$ \\
$p=.48$ & $p=.006$ & $p=.15$ \\
$n=122$ & $n=122$ & $n=122$ \\
\hline
\end{tabular}


El consumo de drogas y el tratamiento en pacientes que realizan actividades dilictivas

Por otro lado, cuando profúndizamos en el seguimiento, esa relación se diluye, de manera que a los doce y dieciocho meses los coeficientes de correlación pierden su significatividad, a excepción del consumo de "otras drogas" que tiende a ser mayor. Esto es debido a que muchos pacientes, en nuestro caso un $28 \%$, desvían sus consumos hacia sustancias menos peligrosas, más toleradas socialmente, de fácil acceso y más baratas (alcohol, hachís, fármacos, etc.); con ello pierde fuerza el binomio actividad delictiva y consumo de opiáceos.

Posteriormente, se realizaron distintos análisis de varianza con el fin de conocer si existían diferencias entre los tres niveles de la variable actividad delictiva previa (ausencia de actividad delictiva, actividad inferior a la media y actividad superior a la media o alta) con relación al consumo de heroína, de "otras drogas" e IGA referidos a los pases de los seis, doce y dieciocho meses. Los resultados mostraron que solo existen diferencias significativas entre los tres niveles del factor con relación al consumo de heroína e IGA a los seis meses, pero no para el resto del seguimiento, en el que las diferencias pierden su significatividad, con ellos se confirma lo señalado anteriormente (Cuadro 4).

\section{Cuadro 4}

Implicación delictiva previa y resultados del tratamiento

\begin{tabular}{|c|c|c|}
\hline \multicolumn{3}{|c|}{ Actividad delictiva previa } \\
\hline Consumo heroína & Consumo "otras drogas" & IGA \\
\hline \multicolumn{3}{|c|}{6 meses } \\
\hline $\begin{array}{c}F_{2,1+12}=\mathbf{3 . 6 3} \\
p=.02\end{array}$ & $\begin{array}{c}F_{2,142}=2.18 \\
p=.11\end{array}$ & $\begin{array}{c}F_{2,148}=5.05 \\
p=.00\end{array}$ \\
\hline & 12 meses & 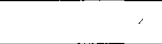 \\
\hline $\begin{array}{c}F_{2.120}=2.24 \\
p=.11\end{array}$ & $\begin{array}{c}F_{2,120}=0.12 \\
p=.88\end{array}$ & $\begin{array}{c}F_{2,120}=0.40 \\
p=.67\end{array}$ \\
\hline \multicolumn{3}{|c|}{18 meses } \\
\hline $\begin{array}{c}F_{2, H 19}=1.00 \\
p=.36\end{array}$ & $\begin{array}{c}F_{2,119}=0.86 \\
p=.42\end{array}$ & $\begin{array}{c}F_{2,119}=1.41 \\
p=.24\end{array}$ \\
\hline
\end{tabular}


Para determinar la dirección de las diferencias del pase de los seis meses comparamos los dos grupos extremos (sin implicación delictiva y con la mayor implicación) mediante una prueba $t$ de Student y con relación al consumo de heróna e IGA, mostrándose los dos grupos diferentes $(t=-2.84, p=.00$ y $t=3.20, p=.00$, respectivamente); por el valor de sus medias, los pacientes que no han desarrollado actividad delictiva previa al tratamiento consumen menos heroína y alcanzan puntuaciones de mayor adaptación en el IGA. Así pues, la actividad delictiva parece tener cierto peso e influencia en la fase correspondiente al tratamiento, ya que después de esta las diferencias tienden a desaparecer por lo que su influencia sería menor y carente de significatividad.

\section{Conclusiones}

- Según las exploraciones realizadas en relación con la actividad delictiva, los pacientes de nuestra muestra pueden agruparse en dos grupos: para uno de ellos la actividad delictiva sería previa o coincidente con el inicio del consumo de sustancias; ello no excluye que llegado el caso y para algunos de estos sujetos la actividad delictiva se instrumentalice al servicio de la drogadicción. Posiblemente, en estos sujetos se darían condiciones para hablar de un evidente desajuste social cuyas manifestaciones más evidentes serían la comisión de delitos y el consumo de drogas. El otro grupo estaría integrado por aquellos pacientes a los que el hábito del consumo les ha arrastrado a la actividad delictiva después de agotar otros recursos y obligados por las necesidades que la dependencia origina de manera que la comisión de delitos sería una consecuencia del consumo de drogas.

- El contacto del paciente adicto con la institución terapéutica supone un significativo descenso en la comisión de delitos; este descenso continúa de manera progresiva a lo largo del seguimiento. Esta reducción no solo es debida a la disminución o abstinencia en 
El consumo de drogas y el tratamiento en pacientes que realizan actividades dilictivas

el consumo, sino también una acción directa del tratamiento en cuanto conlleva una estructuración más adaptativa de la vida del paciente, derivada de la estancia o internado en el centro, de las actividades de ocupación formativa, de ocio, etc.

- La actividad delictiva acumulada por los pacientes antes de iniciar el tratamiento es predictor del consumo de sustancias en el momento de acudir al centro; si bien el valor es bajo, alcanza cierta significatividad estadística. De manera que un grupo de pacientes sufraga el consumo con prácticas delictivas.

- Sin embargo, no todos los pacientes tendrían supeditada dicha práctica al consumo de drogas. La relación entre actividad delictiva previa y búsqueda de sensaciones apunta a un factor ligado al carácter o personalidad que iría más allá, incluso englobaría el propio consumo de sustancias, como una conducta más ligada a un carácter o rasgo de personalidad desinhibido. Por eso los pacientes con puntuaciones más altas en la Batería de Búsqueda de Sensaciones (BBS) tienen un inicio más temprano en el consumo de algunas sustancias, especialmente con cannabis. El inicio en la actividad delictiva podría desmarcarse y sería independiente, en mayor o menor medida, de las necesidades exclusivamente derivadas del consumo de sustancias.

- La severidad de la actividad delictiva previa al tratamiento parece ser un handicap que dificulta o frena la acción terapéutica, ya que los pacientes con mayor historial tienden a cosechar resultados más pobres en el tratamiento. Sin embargo, este hecho corresponde a la respuesta inicial al tratamiento, ya que en la etapa posterapéutica no aparecen diferencias significativas entre pacientes en función de la mayor o menor severidad de sus historiales delictivos. A ello contribuye el desvío del consumo hacia sustancias de mayor tolerancia social, de fácil acceso y más baratas. 


\section{Referencias}

Alemi, D., Stephens, R., Llorens, S. y Orris, B. (1995). A review of factors affecting treatment outcomes: Expected Treatment Outcome Scale. American Journal Drug and Alcohol Abuse, 21, 483-509.

Anglin, M. D. y Speckart, G. (1988). Narcotics use and crime: a multisample multimethod analysis. Criminology, 26, 197-233.

Byquist, S. (1999). Criminality among female drug abusers. Journal of Psychoactive Drugs, 31, 353-363.

Dembo, R., Williams, L., Getreu, A., Genung, L., Schmeidler, J., Berry, E., Wish, E. y Lavoie, L. (1991). A longitudinal study of the relationships among Marijuana/Hashish use, cocaine use and delinquency in a cohort of high risk youths. Journal of Drug Issues, 21, 271-312.

Gerstley, L., Alterman, A., McLellan, A. T. y Woody, G. (1990). Antisocial personality disorder in patients with substance abuse disorders: A problematic diagnosis? American Journal Psychiatry, 147, 173-178. González, M., Ibáñez, I. y Peñate, W. (1997). Consumo de alcohol, búsqueda de sensaciones y dimensiones básicas de la personalidad. Análisis y Modificación de Conducta, 23, 385-404.

Hanlon, T., Nurco, D., Kinlock, T. y Duszynski, K. (1990). Trends in criminal activity and drug use over an addiction career. American Journal Drug and Alcohol Abuse, 16, 223-238.

Jamoulle, P. (2000). Trajectoires judiciaries des usagers de drogues et precarisation. Interventions, 75, 32-38.

Joe, G., Chastain, R., Marsh, K. y Simpson, D. (1990). Opioid addiction and treatment: A 12 years follow-up. Malabar: FL Krieger Publishing.

Judson, B. y Goldstein, A. (1982). Prediction of long-term outcome for heroin addicts admitted to a methadone maintenance program. Drug and Alcohol Dependence, 10, 383-391.

Kandel, D. B. (1978). Longitudinal research on drug use. Empirical finding and methodological issues. Washington DC: HemisphereWiley. 
El consumo de drogas y el tratamiento en pacientes que realizan actividades dilictivas

Luengo, A., Otero, J. M., Romero, E. y Gómez, J. A. (1996). Efectos de la necesidad de búsqueda de sensaciones sobre la involucración en el consumo de drogas de los adolescentes. Análisis y Modificación de Conducta, 22, 683-708.

Pérez Fernández, E. (1990). Aproximación a las relaciones entre conductas adictivas y delincuencia. Comunidad y Drogas, 16, 3949.

Ramssay, M. y Sondhi, A. (2000) Criminal justice implications of drug use by young people. Criminal Justice Matters, 41, 2223.

Roelof, J. (1986, octubre). ¿Debe ser igual la respuesta del sistema de justicia penal ante un delincuente drogadicto que ante otro que no lo es? Ponencia presentada en el Simposium sobre toxicómanos sometidos a un proceso penal, Estrasburgo.

Sánchez Carbonell, J., Brigós, B. y Camí, J. (1989). Evolución de una muestra de heroinómanos dos años después del inicio del tratamiento: Proyecto EMETYST. Medicina Clínica, 4, 135139.

Sánchez Carbonell, J., Camí, J. y Brigós, B. (1988). Follow up of heroin addicts in Spain (EMETYST Project). Results 1 year after treatment admission. British Journal of Addiction, 12, 14391448.

Segest, E., Mygind, O. y Bay, H. (1989). The allocation of drug addicts to different types of treatment. An evaluation and a two-year follow-up. American Journal Drug and Alcohol Abuse, 15, 41-53.

Zuckerman, M. (1979). Sensation seeking: Beyond the optimal level of arousal. Hillsdale: Erlbaum. 